\title{
Un estudio de caso clínico de sobrecarga emocional por conflicto familiar
}

\section{A case report of emotional overload due to a family conflict}

\author{
Laura Torres Moreno ${ }^{1}$, Rut Mesón Torets ${ }^{1}$ y Montserrat Montaño Fidalgo ${ }^{2}$ \\ ${ }^{1}$ Universidad Internacional Menéndez Pelayo, España \\ ${ }^{2}$ Centro de Psicología Álava Reyes, España
}

\begin{abstract}
Resumen: Se expone el estudio de caso clínico de una mujer de 18 años, con sobrecarga emocional por conflicto familiar. Los graves conflictos generados en la convivencia con la mujer de su padre incluyen episodios de violencia física hacia la paciente, falta de apoyo emocional por parte de su padre, la adquisición del rol de cuidadora de su hermano pequeño, así como otras tareas domésticas. Estos factores han favorecido la aparición de un sentimiento de sobrecarga emocional e indefensión en la paciente que ha dificultado cualquier intento de cambio. Se ha aplicado terapia cognitivo-conductual, basada en técnicas de psicoeducación, control y manejo de la ansiedad, entrenamiento en respiración diafragmática, y en comunicación asertiva. Tras la intervención se observa una amplia mejoría reduciendo los niveles de ansiedad y depresión, y aumentando las habilidades sociales y la asertividad para afrontar los conflictos.

Palabras clave: estudio de caso clínico, sobrecarga emocional, terapia cognitivo-conductual

Abstract: The clinical case of an 18-year old woman with signs of emotional overload due to a family conflict is discussed. She reported serious conflicts with her father's wife, including physical violence towards the patient, as well as lack of emotional support from the father, assumption of the role of caregiver of her younger brother and other household chores. These factors resulted in a feeling of emotional overload and helplessness that hindered any attempt to change. Cognitive-behavioural therapy based on psychological education, control, and anxiety management techniques, diaphragm breathing, and assertive communication, was implemented. After the intervention, a
\end{abstract}

Para citar este artículo: Torres Moreno, L. Mesón Torets, R. y Montaño Fidalgo, M. (2021). Un estudio de caso clínico de sobrecarga emocional por conflicto familiar. Revista Clínica Contemporánea, 12(1), Artículo e4. https://doi.org/10.5093/cc2021a4 
clear improvement was seen: levels of anxiety and depression decreased, and social skills and assertiveness to cope with conflicts increased.

Keywords: case report, emotional overload, cognitive-behavioural therapy

Por un lado, y a pesar de no constituir un trastorno mental en sí mismo, la sobrecarga emocional genera un impacto negativo en la calidad de vida de quien la padece, siendo común en personas con un rol de cuidador, ejercido, en su mayoría, por mujeres. Entre los síntomas que se experimentan destacan el nerviosismo y la preocupación, soledad, dificultad para dormir y un bajo estado de ánimo, que se manifiesta, sobre todo, en la pérdida de momentos gratificantes (Prieto, 2013). Estas mismas características se pueden apreciar en el Síndrome de Burnout, caracterizado por la dificultad de afrontar el estrés emocional, agotamiento o sobrecarga emocional, afectando a distintas áreas en la vida de una persona (Aceves et al., 2006). Aunque normalmente, la sobrecarga emocional y el Burnout se asocia al ámbito clínico y laboral, consideramos que en el caso que presentamos a continuación puede asemejarse a la problemática de la paciente debido al rol de cuidadora que ejerce en su ámbito familiar, así como la asunción de todas las responsabilidades domésticos que se detallarán más adelante. En este tipo de situaciones una parte fundamental de la intervención será aprender a aceptar la realidad de aquello que no podemos controlar ni cambiar y a gestionar las emociones que estas situaciones nos generan, trabajar en nuevos hábitos de comunicación y no descuidarse fomentando la aparición de nuevos objetivos que sean motivantes para la persona (Prieto, 2013).

Por otro lado, y a pesar de que cada vez hay una mayor concienciación sobre la violencia ejercida contra los menores, los datos actuales nos dicen que continúa siendo una lacra social, dado que, según los datos ofrecidos por el Ministerio de Interior (2019) en 2018 hubo un total de 6532 menores de 18 años víctimas de violencia familiar. Además, la violencia contra un menor esta incluso menos justificada que cualquier otro tipo de violencia debido a las características del tipo de población como es su dependencia a sus padres, a familiares, figuras de autoridad, etc. Debido a que un menor se encuentra en pleno desarrollo, la violencia genera un factor estresante que altera su desarrollo normal (Nunes y Sales, 2016). El término de violencia familiar hace referencia a cualquier abuso físico, sexual o psicológico que se da entre los miembros de una misma familia. La exposición a este tipo de violencia genera un riesgo en el bienestar de los menores, y un modelo de aprendizaje de conductas violentas dentro del hogar, que provoca un gran impacto negativo en su posterior desarrollo. Entre las consecuencias asociadas, destacan un mayor número de conductas agresivas, sentimientos de indefensión y miedo, una menor competencia social y bajo rendimiento académico, así como, altos niveles de ansiedad y depresión, e incluso pueden llegar a aparecer síntomas traumáticos (Patró y Limiñana, 2005). Además, aumenta la probabilidad de que los menores ejerzan violencia de manera ascendente, es decir, de hijos a padres creando así un ciclo negativo de interacciones sociales, que llevan a una percepción de la sociedad basada en la hostilidad, la inadaptación social y el desarrollo de conductas violentas (Contreras y del Carmen Cano, 2016). Por ello, son esenciales los apoyos sociales y familiares durante la infancia y la adolescencia, para generar vínculos que constituyan elementos imprescindibles en el bienestar de las personas, siendo la adolescencia una etapa especialmente sensible a este respecto. Además, se ha observado que la ventilación emocional en estos casos es esencial, siendo necesarios apoyos sociales para cubrir esta necesidad, y normalizar determinados aspectos (Orcasita y Uribe, 2010).

En el presente trabajo, se exponen las consecuencias de una situación familiar conflictiva, en el estado psicológico de una adolescente, así como la intervención realizada, con el objetivo de normalizar la vida de la paciente, y asegurar su integridad física. A pesar de que la paciente ha alcanzado la mayoría de edad poco antes de iniciar el tratamiento, se ha contextualizado como un caso de maltrato psicológico infantil ya que la situación problemática lleva ocurriendo desde hace un par años y dadas las características sociales y económicas de la paciente. 


\section{Identificación del paciente}

La paciente, R., es una mujer de 18 años. Vive con su padre, su hermano un par de años menor que ella, y C., la pareja de su padre. Original de un país de América Latina, lleva dos años en España. El resto de su familia vive en su país de origen. Estudia para obtener el graduado escolar y realiza un curso de formación profesional en un centro de formación juvenil, donde solicita apoyo psicológico para afrontar su situación familiar. Cabe destacar que algunos datos han sido modificados u omitidos (como por ejemplo nombres, edades, etc) con el objetivo de respetar la confidencialidad de la paciente.

\section{Análisis del motivo de consulta}

Acude a consulta debido a la preocupación y el malestar que está experimentando desde hace dos años, cuando llega a España, por las fuertes discusiones y problemas de convivencia que tiene con C., la mujer de su padre. Esta ejerce principalmente un maltrato psicológico sobre ella, e incluso han llegado a producirse situaciones de violencia física. Refiere sentirse sola, sin apoyos emocionales, y obligada a encargarse de todas las tareas domésticas y el cuidado de su hermano pequeño. Su padre trabaja en un negocio familiar, y C. como asistente del hogar, en cualquier caso, ninguno colabora en las tareas y tampoco le asignan ninguna al hermano pequeño por ser un chico. Verbaliza sentirse atrapada y tratada como una "chacha", y reprendida cuando intenta expresar sus sentimientos al respecto por negarse a hacer lo que le corresponde como "mujer adulta que es". Acepta el mandato de género que su padre le impone a pesar de no estar de acuerdo, pues teme decepcionarle, y al mismo tiempo, evita nuevos conflictos con C., en un intento de frenar nuevos episodios de violencia.

\section{Historia del problema}

La paciente es obligada a emigrar a España hace dos años, con su padre y su hermano pequeño, buscando mayores oportunidades sociales y laborales; decisión apoyada por su madre. Desde el primer momento recibe el rechazo de C., teniendo graves conflictos en la convivencia, incluidas algunas agresiones físicas ejercidas por parte de C. La paciente ha mantenido siempre un estilo de afrontamiento pasivo, excepto en una ocasión, en la cual, ante una agresión física de C., responde violentamente. Refiere haber manifestado querer volver a su país, pero su padre no lo permite dado que la necesita para cuidar de su hermano pequeño, prometiéndole que pronto finalizará su relación con $\mathrm{C}$. Ha encontrado apoyo en una mujer con la que su padre mantiene una relación extramatrimonial. La paciente normaliza esta situación, pues refiere haber ocurrido también durante la relación de sus padres, puntualizando que su padre es "mujeriego" y posee una ideología machista. Se siente sola y aislada, pues cuidar de su hermano pequeño y ser "una mujer de su casa" impiden que participe en actividades de ocio y generar relaciones interpersonales sólidas. Su preocupación por no experimentar nuevos episodios de violencia ha hecho que evite cualquier intento de cambio, generando un sentimiento de indefensión. Intenta pasar en casa el menor tiempo posible cuando C. se encuentra ahí, y cuando coinciden, permanece en su habitación para evitarla, aunque a menudo acaban discutiendo. La paciente interpreta los conflictos con C. como ataques personales hacia ella, y al evitarlos, se considera débil. Tiene muchas dificultades a la hora de negarse a hacer lo que otras personas quieren, por miedo a las consecuencias que pueda tener expresar su opinión, dado que no quiere hacer sentir mal a los demás, aceptando así las altas exigencias de su padre.

\section{Análisis y descripción de las conductas problema}

La principal conducta problema son los altos niveles de ansiedad, el bajo estado de ánimo y el sentimiento de indefensión. Esta situación está provocada por el conflicto familiar asociado principalmente al maltrato psicológico por parte de C. Estas conductas problema están moduladas por el miedo de R. a la desaprobación de 
los demás, principalmente del padre, el déficit que presenta en asertividad y control de la ansiedad, el comportamiento de C., y el funcionamiento de su padre. Este a su vez genera altos niveles de exigencia, y la asunción de un alto número de responsabilidades, entre las que destaca el rol de cuidadora de su hermano pequeño. Todo su contexto social y familiar, está influenciado por su cultura de origen, que afianza estas creencias machistas. Además, se añade la falta de apoyo emocional, el deficiente contexto social, y la falta de recursos económicos para independizarse. Todos estos factores han llevado a R. a padecer una elevada sobrecara emocional que está provocando un impacto negativo en su vida. No obstante, cuenta con dos factores de protección: la pareja en la relación extramatrimonial de su padre, a quien recurre para no pasar tiempo en casa y a quien considera una amiga, y el centro de formación, donde encuentra un lugar en el que evadirse, y la ayuda de profesionales especializados.

Se establece entonces una hipótesis de origen en la cual, el hecho de emigrar de su país hace que por un lado pierda reforzadores positivos que disfruta allí, y no encuentra en España, como pueden ser amigos, familia, etc. Por otro lado, hay un aumento de estimulación aversiva por los conflictos con C., y el exceso de tareas domésticas. El comportamiento de esta no es contingente ante la conducta de R., por lo tanto, no lo puede predecir o evitar, generando así un sentimiento de indefensión y pasividad, que desembocan en un bajo estado de ánimo y una importante reducción de su nivel de actividad. En cuanto a las tareas domésticas, el hecho de no cumplirlas genera un castigo positivo, siendo éste la crítica paterna. Su historia de aprendizaje ha condicionado su deseo de agradar a los demás, por lo que supone un refuerzo ajustarse a lo que los demás demandan. De esta manera, se ajusta a las expectativas de su padre, cargando con todas las tareas, reduciendo su contexto social y de ocio, y generando un bajo estado de ánimo, así como la disminución de su actividad social.

El problema se mantiene porque sigue en el mismo contexto y se mantienen los mismos procesos de aprendizaje. Además, a los factores de origen que también intervienen en el mantenimiento se añaden sus pensamientos sobre la interpretación de la situación, del tipo "no me respetan", "soy débil", "va a por mî", generando constantemente el mismo sentimiento de malestar. Otro factor importante que favorece el mantenimiento del problema es una constante búsqueda de aprobación, a pesar de que ésta no es contingente, y que le genera

Figura 1. Modelo explicativo del mantenimiento del problema

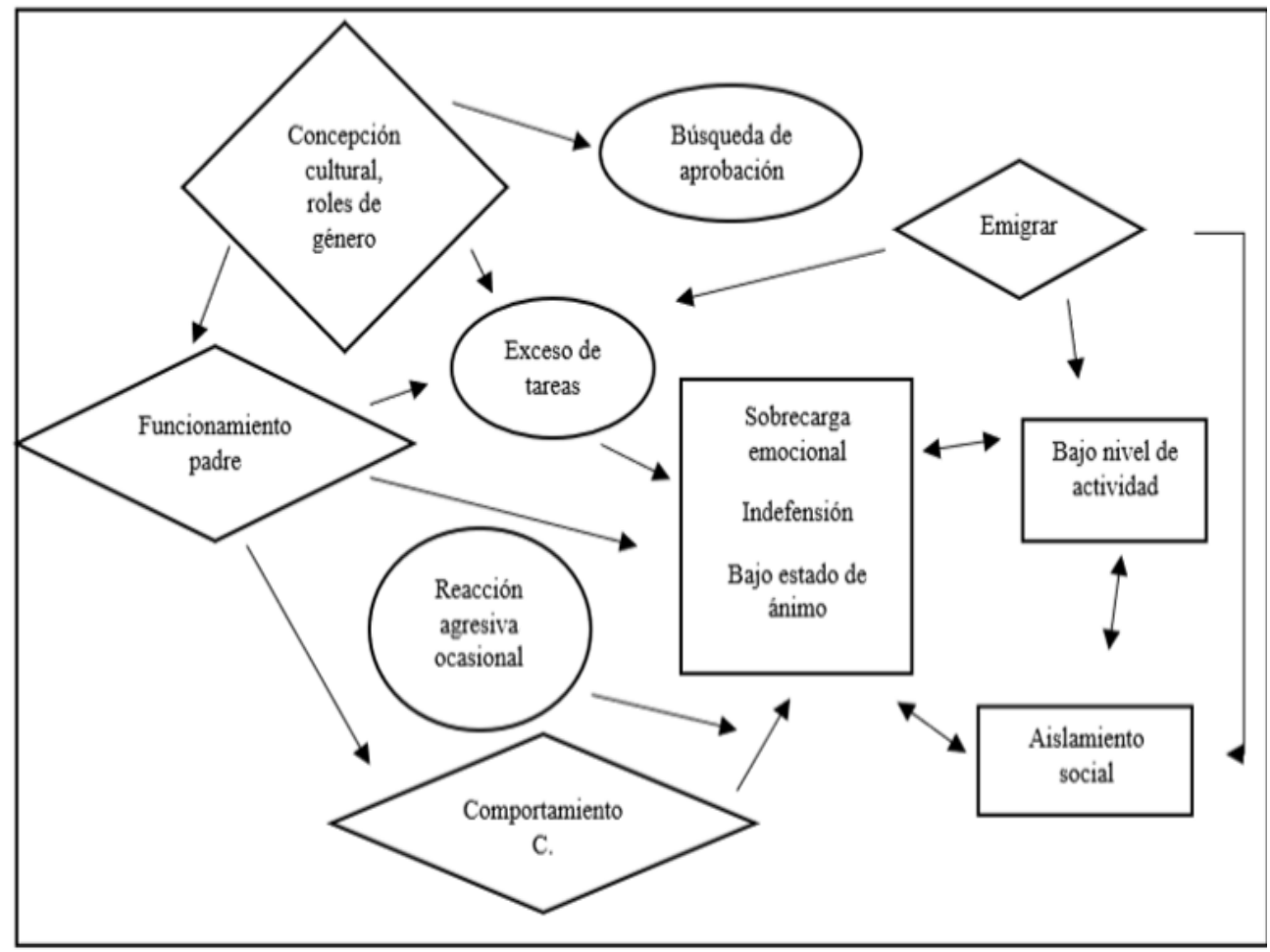


altos niveles de autoexigencia, y un mayor número de tareas. A pesar de sus esfuerzos, R. no consigue evitar la mayoría de los estímulos temidos, y aparece una respuesta de indefensión, generando más malestar y mayor pasividad a la hora de buscar otras alternativas y fuentes de gratificación. Por ello, la baja tasa de reforzadores, debida al exceso de tareas, al bajo estado de ánimo, y a emigrar y cambiar de contexto, influyen en el estado de ánimo que presenta la paciente. Para favorecer la compresión del mantenimiento del problema en la paciente, a continuación, se incluye el modelo explicativo en la Figura 1.

\section{Establecimiento de las metas del tratamiento}

Los objetivos planteados junto a R. fueron los siguientes: (1) garantizar la integridad física, (2) mejorar el manejo de los conflictos con C., y (3) aumentar el estado de ánimo y reducir ansiedad.

\section{Estudio de los objetivos terapéuticos}

Primero se garantiza su integridad física, ofreciéndole pautas en caso de sentir que se encuentra en peligro. Para mejorar la gestión del conflicto y reducir el impacto emocional, se trabajó el control de la ansiedad generada mediante técnicas de respiración diafragmática, y se entrenó a $\mathrm{R}$. en técnicas de comunicación asertiva. Además, una parte fundamental de la intervención se centró en no entrar a provocaciones de C. reestructurando las interpretaciones negativas que surgían por un lado de la evitación del conflicto, y por otro de responder a este en algunas ocasiones, generando así una mayor aceptación de la situación. Para mejorar su estado de ánimo, trasladamos la importancia de aumentar el número de actividades gratificantes que realizaba R., así como ampliar su círculo social. Para ello, se operativiza qué actividad haría cada fin de semana, y con quién, procurando que los planes establecidos fueran llevados a cabo con diferentes personas. Trabajando estos aspectos buscamos empoderar a R., con el objetivo de salir de la indefensión que siente.

\section{Selección del tratamiento más adecuado}

Se trabaja mediante el modelo cognitivo conductual, con psicoeducación, entrenamiento en técnicas de respiración diafragmática, y en comunicación asertiva, con el objetivo de empoderarla y reducir el sentimiento de indefensión. Comprobamos que R. no presenta ningún trastorno recogido en los manuales vigentes de salud mental, pero sí síntomas de sobrecarga emocional. A pesar de que la literatura científica está más destinada al rol del cuidador en el ámbito clínico y laboral, sí que se han establecido algunos métodos para su tratamiento. Por ello, enfocamos la intervención en la aceptación de la situación, y el manejo de emociones (Prieto, 2013). Nos centramos en las técnicas expuestas anteriormente, pues el entrenamiento en técnicas de relajación, asertividad, afrontamiento, y las técnicas de autocontrol, podrían reducir la sobrecarga emocional generada, al igual que ocurre en el Síndrome de Burnout (Ortega y López, 2004). La eficacia de la terapia cognitivo conductual, favorece una mejoría a nivel emocional, tanto del estado de ánimo como de la ansiedad (Hollon et al., 2002). Por ello, se ha trabajado mediante reestructuración cognitiva aquellos pensamientos distorsionados y causantes de indefensión y desesperanza, y con activación conductual el aumento de las actividades gratificantes.

\section{Selección y aplicación de las técnicas de evaluación y resultados obtenidos en esta fase}

En un primer momento se utilizó la entrevista clínica no estructurada recopilando datos acerca del origen del problema, y formulando una posible hipótesis de mantenimiento. Se utilizaron a su vez autorregistros para obtener ejemplos de las situaciones de conflicto como vemos en la Tabla 1. 
Tabla 1. Registro de situaciones que generan malestar

\begin{abstract}
Situación
Papi me llama por teléfono porque C. le ha dicho que tengo la música muy alta.

Me voy con la novia de papi. Bebo una cerveza y al llegar a casa papi me riñe.
\end{abstract}

Mi padre llega a casa y me riñe porque no tengo los platos fregados.
Qué siento

Incómoda

Enfado con C

Impotencia

Incomodidad

Presión en el pecho

Enfado

Me cuesta respirar

Dolor en la cabeza

Me siento obligada

Nerviosa
Qué pienso

"Me tienen harta"

"Darle una galleta"

"La voy a agarrar por el cuello"

"Algo le ha dicho C. para que me hable asî́"

"Pienso que me enerva"
Qué hago

Primero apago la música, y luego la vuelvo a poner

Me voy a dormir 


\section{Sesión 2}

Continúa la evaluación y recogida de información, psicoeducación en asertividad, y mandamos registro de situaciones que le generan malestar. Trabajamos pautas de comunicación, evaluando su repertorio y ofreciendo diferentes alternativas. Tratamos la gestión del tiempo. Valoramos el riesgo de la relación con C., y damos pautas de seguridad para que evite el conflicto, garantizando su seguridad física.

\section{Sesión 3}

Observamos una leve mejoría en el estado de ánimo. No trae las tareas propuestas por lo que se lleva a cabo el registro en la sesión. Hacemos hincapié en la importancia que tiene la realización de las tareas, y volvemos a mandar los registros para la semana siguiente. Observamos un discurso circular en cuanto a la relación con C., que dificulta cambiar el foco de atención respecto al problema familiar.

\section{Sesión 4}

Acude contenta, aunque ha tenido discusiones durante la semana. Se establece la estructura que llevarán en adelante la sesiones. Dado el discurso circular, se establecen los primeros 15 minutos de la sesión como desahogo emocional, generando un espacio de apoyo y protección y el resto del tiempo, de trabajo. La paciente acepta este nuevo cambio. Esta decisión supone un punto de inflexión en la terapia, provocando en R. una amplia mejoría. Se realiza el registro de situaciones, y trabajamos la identificación de respuestas fisiológicas y emocionales, e introducimos entrenamiento en técnicas de respiración ante los conflictos con C. Destacamos la importancia de aumentar su círculo social y actividades de ocio, operativizando cómo, cuándo, y dónde se realizarán. Mantenemos como tarea el registro de situaciones y pasamos cuestionarios de estado de ánimo y ansiedad.

\section{Sesión 5}

Tras un fuerte conflicto con C., la paciente verbaliza haber sentido ira y desesperación, y teme llegar a perder el control y protagonizar de nuevo un episodio de violencia física. Decide abandonar la vivienda, pasando el fin de semana fuera de casa. Reforzamos la decisión tomada, dejando clara la importancia de mantener su seguridad en todo momento, sin exponerse a nuevas agresiones físicas, recogiéndola a nivel emocional, y empatizando con ella en todo momento. Recordamos que dispone del entrenamiento en respiración para rebajar la ansiedad. El plan de actividades realizado durante la sesión anterior había sido puesto en marcha, pero queda anulado tras el conflicto, por lo que lo recuperamos para ser llevado a cabo durante el fin de semana. Finalmente, mantenemos el registro de situaciones que generan malestar.

\section{Sesión 6}

Apreciamos mejoría tras pasar una semana tranquila. Reforzamos poner en práctica las técnicas de respiración, trabajando así el autocontrol. Aprovechando una visita familiar en casa, sale con amigos. Valoramos su esfuerzo por realizar actividades gratificantes, demostrando así que, tras sacar tiempo para ella, se encuentra mejor. Planificamos nuevo fin de semana. Descartamos el autorregistro dado que, a pesar de haber habido conflicto, su impacto ha sido menor, y lo posponemos para la siguiente sesión en caso de que el impacto vuelva a ser mayor. Se produce un importante cambio en esta sesión, y asimila la propuesta presentada de centrarse en lo que ella pueda hacer. Acepta que su padre y C, muy probablemente no cambiarán, cambiando así el foco de atención desde lo externo y ajeno a su control, a lo propio y manejable.

\section{Sesión 7}

Semana tranquila al poner en práctica las técnicas explicadas. Realiza planes diferentes a los organizados y afirma pasarlo bien, por lo que lleva a cabo actividades gratificantes lo que valoramos muy positivamente. Trabajamos técnicas de asertividad y comunicación, y acordamos diferentes situaciones a las que tiene que enfrentarse como tarea. Además, nos ofrecemos a ayudarla con la realización de su CV y una carta de presentación, pues intentará encontrar trabajo para poder viajar en verano y visitar a su familia. 


\section{Sesiones 8 y 9}

Trabajamos técnicas de comunicación, y valoramos que continúe poniendo en práctica las técnicas anteriormente trabajadas, afianzando los logros conseguidos hasta ahora. Dejamos al margen la realización del CV ya que S., decide no buscar trabajo y esperar a terminar el curso. Tras hablarlo con ella y valorar sus razones, reforzamos su capacidad a la hora de tomar decisiones.

\section{Seguimiento}

Las sesiones de seguimiento se establecieron tras la sesión nueve y pasaron a ser sesiones quincenales con el objetivo de observar que los cambios obtenidos se mantienen en el tiempo de manera estable, y que no sólo se deben a cambios favorables en el contexto (como por ejemplo visitas en la casa). Por lo tanto, el foco de atención a partir de este momento se centró en afianzar lo aprendido y generalizarlo a otros contextos, de tal manera que la paciente se sienta empoderada en futuras situaciones conflictivas, no sólo familiares, y tenga las herramientas necesarias para hacerlas frente. Durante esta fase, se realizó una evaluación post tratamiento, comprobando que se habían alcanzado los objetivos propuestos y se habían bajado los niveles de ansiedad y depresión concluyendo así la intervención.

\section{Evaluación de la eficacia del tratamiento y conclusiones}

Tras observar los resultados obtenidos de la intervención, mediante cuestionarios y observación directa, se puede ver una mejoría en la paciente. Es capaz de controlar mejor las emociones generadas por los conflictos y ha aprendido a gestionar la ansiedad que estos le producen. Además, gracias al entrenamiento en técnicas de asertividad, es capaz de comunicarse en relación con determinados temas que, previamente a la intervención, prefería evitar por miedo a la desaprobación de los demás. Cabría destacar cómo R. justificaba el comportamiento de su padre, e incluso su pasividad y cómo a lo largo de las sesiones ha sido capaz de reconocer el daño emocional que esta situación le ha generado, y lo ha hecho expresando, sin miedo, todo lo que ha significado para ella no haber contado con el apoyo paterno. Verbaliza encontrarse anímicamente mejor, sintiéndose más empoderada a la hora de enfrentar los conflictos. Refiere contar con más estrategias para afrontar todas aquellas situaciones que le generan malestar, y esto se ve reflejado en la interpretación que realiza ahora sobre su situación familiar, y en cómo repercute en su manera de afrontarla. Además, es consciente de que evitar el conflicto es la mejor opción, a la espera de poder independizarse, cambiando la interpretación que realizaba, pues el hecho de evitar enfrentamientos hacía que se sintiese débil. Para comprobar que los niveles de ansiedad y depresión son mejores que al inicio del tratamiento, llevamos a cabo una evaluación post intervención al final de la fase de seguimiento como se indicó anteriormente. Por ello, observamos que estos niveles disminuyen obteniendo las siguientes puntuaciones; en el $B D I$ obtienen una puntuación directa de1, encontrándose entre 1 y 10 en un estado normal. En el $B A I$ obtiene una puntuación de 1, siendo entre 0 y 7 un estado mínimo de ansiedad. Y en el STAI obtiene una puntuación de 5 en ansiedad Estado encontrándose en un percentil 4. En el caso de la ansiedad Rasgo, la puntuación obtenida es de 15 indicando esta un percentil de 17. Para una mayor comprensión, se muestra a continuación una representación gráfica en la Figura 2 de las puntaciones obtenidas en las evaluaciones pre-post.

Para finalizar, y tras la realización del caso clínico, se puede observar la eficacia de los métodos cognitivos-conductuales, contrastando así la evidencia científica. En este caso, uno de los factores claves del éxito de la intervención fue el uso de la reestructuración cognitiva, provocando un cambio en la interpretación de la paciente que la focaliza en su propio bienestar. Este cambio le permitió dejar de lado la desaprobación paterna al no cumplir con sus exigencias y centrarse en sí misma. También se pone de manifiesto la importancia de promover una buena alianza terapéutica, que en el caso que nos ocupa, permitió la ventilación emocional, la creación de un espacio seguro y ejercer como una figura de referencia para la paciente. Estos aspectos permitieron una buena adherencia al tratamiento, asistiendo a todas las sesiones y llevando a cabo las pautas dadas. 
Figura 2. Puntaciones de depresión del cuestionario de Beck y de ansiedad de los cuestionarios de Beck y Rasgo-Estado, pre-tratamiento y post-tratamiento

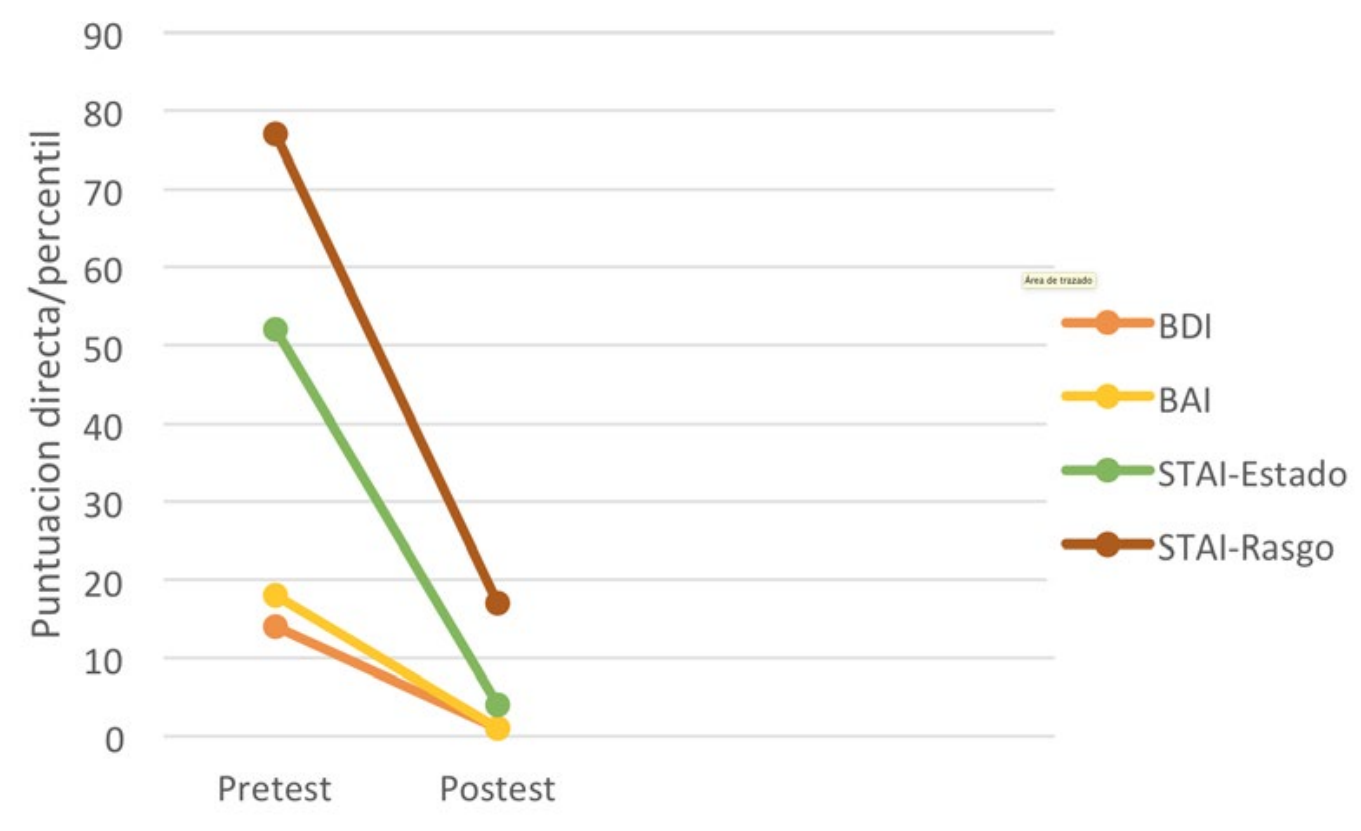

BDI (Beck Depression Inventory); BAI (Beck Anxiety Inventory); STAI (State Trait Anxiety Inventory)

Por último, hay que destacar la importancia de continuar apoyando la investigación científica en general, y en el tema de la sobrecarga emocional dentro del ámbito familiar en particular. La existencia de bibliografía científica es de vital importancia a la hora de llevar a cabo intervenciones permitiendo así la utilización de estrategias avaladas. Es por ello, que se presenta este caso, con el objetivo de ser de utilidad a compañeros en futuras intervenciones con características similares.

\section{Referencias}

Aceves, G., López, M. A., Moreno, S., Farias, F. y Suárez, J. J. (2006). Síndrome de burnout. Archivos de Neurociencias, 11(4), 305-309.

Beck, A. T., Brown, G., Epstein, N. y Steer, R. A. (1988). An inventory for measuring clinical anxiety: Psychometric properties. Journal of Consulting and Clinical Psychology, 56(2), 893-897. https://doi. org/10.1037/0022-006X.56.6.893

Beck, A. T., Steer, R. A. y Brown, G. K. (1996). Manual for the Beck Depression Inventory-II. Psychological Corporation.

Beck, A. T., Ward, C. H., Mendelson, M., Mock, J. y Erbaugh, J. (1961). An inventory for measuring depression. Archives of General Psychiatry, 4(6), 561-571. https://doi/10.1001/archpsyc.1961.01710120031004

Contreras, L. y del Carmen Cano, M. (2016). Child-to-parent violence: The role of exposure to violence and its relationship to social-cognitive processing. The European Journal of Psychology Applied to Legal Context, 8(2), 43-50. https://doi.org/10.1016/j.ejpal.2016.03.003

Hollon, S. D., Thase, M. E. y Markowitz, J. C. (2002). Treatment and prevention of depression. Psychological Science in the public interest, 3(2), 39-77. https://doi.org/10.1111/1529-1006.00008

Ministerio de Interior (2019). Víctimas de maltrato y violencia. Infancia en datos. http://www.infanciaendatos. 
Nunes, A. J. y Sales, M. C. V. (2016). Violence against children in Brazilian scenery. Ciencia \& Saude Coletiva, 21(3), 871-880. https://doi.org/10.1590/1413-81232015213.08182014

Orcasita, L. y Uribe, A. (2010). La importancia del apoyo social en el bienestar de los adolescentes. Psychologia, 4(2), 69-82. https://doi.org/10.21500/19002386.1151

Ortega, C. y López, F. (2004). El burnout o síndrome de estar quemado en los profesionales sanitarios: revisión y perspectivas. International Journal of Clinical and Health Psychology, 4(1), 137-160.

Patró, R. y Limiñana, R. M. (2005). Víctimas de violencia familiar: Consecuencias psicológicas en hijos de mujeres maltratadas. Anales de psicología, 21(1), 11-17.

Prieto, V. (2013). La soledad del cuidador. La Esfera de los Libros.

Sanz, J., Navarro, M. E. y Vázquez, C. (2003). Adaptación española del Inventario para la Depresión de Beck-II (BDI-II): 1. Propiedades psicométricas en estudiantes universitarios. Análisis y notificación de conducta, 29(124), 239-288.

Sanz, J. y Navarro, M. E. (2003). Propiedades psicométricas de una versión española del Inventario de Ansiedad de Beck (BAI) en estudiantes universitarios. Ansiedad y Estrés, 9(1), 59-84.

Artículo recibido: $17 / 06 / 2020$

Artículo aceptado: 18/02/2021 